\title{
NACHBARSPRACHEN UND -REGIONEN: CHANCEN FÜR EINE BESSERE VERSTÄNDIGUNG IN EUROPA
}

\author{
URSULA BOCK \\ Europa-Universität Viadrina - Frankfurt (Oder)
}

\begin{abstract}
The following essay discusses the context of cultural problems and language problems between neighboring countries and regions in Europe. About two thousand years ago Greek and Latin had a lasting influence especially on Western European languages, whereas in the beginning modern era French became the prevailing idiom, which is nowadays removed from English as the language of technology and culture. In addition peoples migrations because of wars and poverty as well as increasing tendencies of globalisation raise the clashes of different languages and cultures all over Europe. Moreover, several European countries, e.g. Belgium, Switzerland or Italy, have to deal with multilingual inhabitants and a lot of cultural and social problems resulting from this situation.

The second part of the essay concentrates on the historical and cultural development along the German-Polish borderline during the last 50 years. Special interest lies on the contemporary situation that is marked by increasing cooperation between the two countries. The improvement of cooperation concerns economy as well as culture and science including the promotion of learning the neighboring language on both sides of the frontier and thus building a new region of mutual understanding.
\end{abstract}

\section{Der Begriff}

Der Begriff Nachbarsprache hat einen historischen ebenso wie einen räumlichen Bezugsrahmen. Beide sollen hier kurz angesprochen werden.

Historischer Kontext: Im Zuge der Ausbreitung des Römischen Reiches nach Norden fand auch eine sprachliche Annäherung des germanischen und romanischen Raumes statt. So gelangten zahlreiche griechisch-römische Sprachpartikel in das Germanische hinein (fenestra - das Fenster; camera-die Kammer u.v.a.). Umgekehrt nahm mit der Völkerwanderung auch das Germanische Einfluß auf die romanischen Sprachen. So geht zum Beispiel das französische $h$ aspiré auf germanischen Einfluß zurück (Wandruszka 1994:14f.). 
Im Mittelalter setzte sich der griechisch-lateinische Wortschatz des Christentums und der Klosterschulen in zahlreichen europäischen Alltagssprachen fest (Beispiel für das Deutsche: angelus - Engel; crux - Kreuz etc.). In ihrer neuen Umgebung haben sich diese Lehenswörter der Landessprache angepaßt. Doch weisen sie vielfach bis heute auf ihren lateinischen Ursprung zurück.

Am Englischen läßt sich die historische Durchdringung der unterschiedlichen Spracheinflüsse besonders gut nachvollziehen. Nach der normannischen Eroberung der Insel (Hastings 1066) vermischte sich das Normannenfranzösisch mit dem Angelsächsischen zu einer neuen gemeinsamen Sprache, die im Humanismus und in der Renaissance um zahlreiche griechisch-römische Einflüsse erweitert wurde (Wandruszka 1994:17). Und nicht erst heute bereichert das Englische selbst die europäischen Sprachen. Diese Entwicklung setzte bereits im 18. Jahrhundert ein, als englische Geselligkeit und Lebensart Eingang in die festländische Kultur fanden.

Insgesamt ist festzustellen, daß viele sprachliche Durchdringungen von einst im Laufe der Zeit fester Bestandteil der jeweiligen Landessprache geworden sind. Diese Entwicklung gegenseitiger Einflußnahme besteht bis heute fort. Als Beispiel dafür mag der inzwischen universal verwandte Ausdruck „Waldsterben" gelten (im Französischen: le Waldsterben). Dieser Begriff läßt übrigens erkennen, daß Nachbarsprachen keinesfalls an lokale Grenzen gebunden sind, sondern über die Medien rasche Verbreitung selbst über Kontinente hinweg finden können. Die globale Vernetzung hat sicherlich die Tendenz zu einer stärkeren Angleichung der Sprachen untereinander verstärkt, und zwar vor allem zugunsten des Englischen. Das läßt sich vor allem in bestimmten Bereichen wie der Kunst und Musik, der Technologie und der Wissenschaft beobachten. Auf diese Weise hat sich eine große Sprachenverwandtschaft in Europa gebildet, so daß Sprachwissenschaftler heute dafür plädieren, den gemeinsamen europäischen Wortschatz für den Fremdsprachenunterricht produktiver zu nutzen und durch die Veranschaulichung der Bezüge das Lernen zu erleichtern (Wandruszka 1994:16).

Räumlicher Kontext: Dem strengen Wortsinne nach handelt es sich bei Nachbarsprachen um Sprachen von Nachbarn. Darunter werden zunächst staatliche Nachbarn verstanden. Dabei ist zu beachten, daß es sich bei dem Terminus Nationalsprache in der Regel um eine Setzung handelt oder zumindest um einen Euphemismus. Denn die Heimatsprache ist vielfach ein regionaler Dialekt von begrenzter Verbreitung. Die regionale Erfahrung prägt ihre Sprecher über eine national verbindende Identität hinaus. Als scheinbar gegenläufige Tendenz wuchs seit dem 19. Jahrhundert mit der Bildung neuer Nationalstaaten in Europa auch die Bedeutung der Nationalsprache als wesentlicher Faktor kultureller Identität. Aus dieser Entwicklung resultierte auch die Festsetzung einer normierten Hochsprache, die in der Schule vermittelt wird und in Deutschland zum 
Beispiel durch den Duden verbindlich festgeschrieben ist. Verstärkt wurde die Tendenz zur Hochsprache dadurch, daß die Mobilisierung der Gesellschaft, die Abwanderung vom Land in die Stadt ebenso wie Prestigefaktoren dazu beigetragen haben, daß regionale Idiome immer mehr ihre Bedeutung verloren haben und teilweise völlig verschwunden sind (Plattdeutsch zum Beispiel). Dennoch haben regionale Idiome in Deutschland in moderierter Form ihren Platz behaupten können. Damit bestehen zwei Strömungen gleichzeitig: der regionale Dialekt auf der einen sowie die verbindliche Hochsprache auf der anderen Seite.

Nachbarsprachen werden auf der nationalen Ebene vor allem auf der Basis der Hochsprache diskutiert und unterrichtet. Gleichzeitig ist festzustellen, daß gerade mit der Erweiterung der Europäischen Union das Schlagwort von dem „Europa der Regionen“ in den letzten Jahren zunehmend Verbreitung gefunden hat. Dieser Begriff bedeutet nichts anderes, als daß regionale Zusammenschlüsse heute auf internationaler Ebene gedacht werden. Als beispielhaft dafür können zahlreiche Euroregionen im deutsch-belgisch-niederländischen und französischen Grenzbereich gelten. Die räumliche Nähe ließ unter dem Patronat der EU zahlreiche gemeinsame Entwicklungen und Kooperationen entstehen, die die Wirtschaftskraft der Regionen entscheidend stützen. Über die nationalen Grenzen hinweg entwickelte sich auch eine sprachliche Verständigung, die an der westlichen Grenze der Bundesrepublik Deutschland auf dem Niederdeutschen, Alemannischen und/oder dem Französischen basiert und gemeinsame Idiome der Menschen beiderseits der Grenzen hat entstehen lassen. Natürlich liegt der Grund für die relativ unproblematische Verständigung in diesen Regionen in einer über lange Zeiträume hinweg relativ homogenen Besiedlung und einem kulturellen Austausch, der selbst zahlreiche territoriale Grenzverschiebungen und zwei verheerende Weltkriege überdauert hat. Darin unterscheiden sich die westlichen Grenzregionen Deutschlands deutlich von ihren östlichen, so $\mathrm{da} ß$ die besonderen historischen und politischen Bedingungen dort einer gesonderten Betrachtung bedürfen.

Sprachenkonflikte können sich entlang staatlicher Grenzen, aber auch innerhalb eines Staates auftun. Beispiele hierfür sind zum einen mehrsprachige Staaten, wie zum Beispiel die Schweiz, Belgien und Luxemburg. Zum anderen aber auch das zunehmend virulent werdende Phänomen der Sprachinseln von Migranten, die sich plötzlich einer Nachbarsprache ausgesetzt sehen, die etymologisch von ihrer Muttersprache weit entfernt ist. In diesen Bereichen kommt der nationalen Sprachenpolitik eine besondere Bedeutung zu. Die Konsequenzen unterschiedlicher Sprachregionen für die Staatengebilde werden bereits seit dem 19. Jahrhundert diskutiert: in den USA, das ein Einwanderungsland für sehr unterschiedliche Sprachgruppen wurde, aber auch in Europa. In jedem Fall bedeutet Sprachenpolitik immer auch Kulturpolitik, und jeder Staat hat die Entscheidung zu treffen, inwieweit er mit der Sprache entweder von Migranten im 
eigenen Land oder von Bevölkerungsminderheiten mit eigener Sprache auch die damit verbundene eigene Kultur zu akzeptieren bereit ist.

\section{Der Kontext: Mehrsprachigkeit und Multikulturalität in Europa}

Die durch die Europäische Gemeinschaft erleichterte Mobilität innerhalb der Union ebenso wie der Zustrom vieler Migranten aus politischen und wirtschaftlichen Gründen, sei es aus Afrika, Asien, der ehemaligen Sowjetunion oder Exjugoslawien wie auch der internationale Tourismus haben die Ausweitung kultureller Vielfalt und Erfahrung zwangsläufig zur Folge. „Die Freizügigkeit in Europa“, schreiben Finkenstaedt/Schröder, „wird keine automatische 'Umbürgerung' in den jeweiligen Gaststaat zur Folge haben... Es bleibt nur die Möglichkeit, durch eine neue Einstellung zum fremdsprachigen Menschen das $\mathrm{Zu}$ sammenleben zu erleichtern. Dazu gehört das sprachliche Aufeinanderzugehen durch einen intensiveren und diversifizierten Fremdsprachenunterricht und eine Intensivierung und Verbesserung des Unterrichts in der Muttersprache für viele Minoritäten in nicht wenigen Ländern Europas" (Finkenstaedt/Schröder 1990:22). Dazu bestehen in den Ländern der Europäischen Union durchaus günstige Voraussetzungen. Denn erstens sind die Staaten durchweg demokratisch und pluralistisch strukturiert. Und zum anderen bestehen starke wirtschaftliche Verflechtungen innerhalb der Union selbst sowie weit über ihre Grenzen hinaus, so daß sich eine Abschottung gegenüber den Nachbarn von vornherein verbietet und statt dessen eine tendenzielle Öffnung nach außen geboten erscheint. Diese Entwicklung hat sich seit dem Ende des Zweiten Weltkrieges im westlichen Europa rasant beschleunigt. Starke Migration aus dem Osten Europas durch erzwungene Umsiedlungen sowie die gewollte Anwerbung von Arbeitskräften vor allem aus ärmeren Regionen des Mittelmeerraumes (Italien, Türkei, Portugal, Spanien, Nordafrika) haben die Begegnung mit zahlreichen neuen Sprachen und kulturellen Eigenheiten zur Folge gehabt. Während die Anpassung der deutschen Migranten nach dem Zweiten Weltkrieg insgesamt eher reibungslos und relativ schnell vonstatten ging, läßt sich gleiches von der Integration der sogenannten Gastarbeiter bzw. Migranten fernerer Kulturen nur sehr bedingt sagen.

Der Grund dafür liegt natürlich zunächst einmal in der sprachlichen und nicht selten auch einer kulturell-religiösen Differenz. Was im Urlaub häufig pittoresk und interessant anmutet, erweckt in der heimischen Nachbarschaft nicht selten Vorbehalte und Ressentiments, über die sich nicht jeder unmittelbar klar ist. Die Frage der Begegnung von Kulturen wird heute vielfach unter dem Stichwort der Identität und der Differenz diskutiert. Aus diesen unmittelbaren 
Erfahrungen geht die Notwendigkeit der Beachtung multikultureller Erwägungen bei der Debatte um die europäische Identität klar hervor. Denn heute tritt das Phänomen der neuen nicht-europäischen Minoritäten in Europa, das sich neben dem immer schon bestehenden Multikulturalismus innerhalb des Kontinents bemerkbar macht, offen zutage. Die bewußte Erinnerung an die eigene multikulturelle europäische Vergangenheit, die auf einer Synthese der griechisch-römischen Antike, des Judentums und des Christentums beruht, sollte hier durchaus hilfreich sein.

Nach Charles Taylor klagen heute Minoritäten zunehmend das Recht auf Entfaltung ihrer Lebensgewohnheiten ein, ihrer Tradition, Gebräuche, Religion. Ihnen genüge die Respektierung ihrer menschlichen Würde bzw. die Anerkennung gleicher Rechte aller Staatsbürger nicht mehr. „Diese 'Politik der universellen Würde', so meinen die Angehörigen von Minoritäten, reiche jedoch nicht aus, die Anerkennung der kulturellen Besonderheiten ihrer Minorität zu garantieren“ (Lützeler 1995:99). Sie fordern daher ein Recht auf Differenz, das die Bewahrung ihrer kulturellen Identität ermöglichen solle. Taylor sieht daher die Berechtigung eines Kompromisses, der auf die Ängste von Minoritäten Rücksicht nimmt und die Wahrung ihrer Identität vorsieht. Allerdings sollten Sonderrechte für Minoritäten nicht zu einer Abschließung dieser Gruppen. Vielmehr müßten besondere Privilegien den kulturübergreifenden Dialog fördern. Taylor bezieht sich dabei auf die kanadischen Gegebenheiten.

Demgegenüber vertritt Jürgen Habermas ein durch die Aufklärung beeinflußtes universalistisches Liberalismusmodell. Universalisten wie Habermas erwarten von Minoritäten die Anerkennung der Verfassung des Landes, in dem sie leben, und die Respektierung demokratischer Werte, aber keine „Akkulturation", das heißt keine ethnisch-kulturelle Anpassung. Im Unterschied $\mathrm{zu}$ Taylor hat Habermas die deutsche Situation der Gegenwart vor Augen. Er sieht vor allem eine Gefahr der Segmentierung, das heißt ein Auseinanderfallen der Gesellschaft insgesamt in unterschiedliche kulturelle Identitäten (Lützeler 1995:100f.; Schiffauer 1997:144ff.). Demgegenüber spricht Wierlacher (Wierlacher 1993:64) davon, kulturelle Fremdheit als Glück und nicht als Unglück menschlicher Existenz aufzufassen, „weil sie ein Stück Freiheit sichert: sie schützt uns paradoxerweise vor Bestimmungen durch andere. Eben dieser Schutz war und ist auch eine der alten Bedeutungen von Gastfreundschaft; in der berührten Dialektik von Fremdheit und Freiheit liegt deren kulturschöpferische Kraft.“

Wissenschaftler wie die genannten fordern eine dialogische Beziehung zwischen den Kulturen. Ethnozentrismus ist nicht gefragt, vor allem auch nach den Erfahrungen rassistisch motivierter Greueltaten in diesem Jahrhundert. Dennoch sind zunehmend auch fundamentalistische Tendenzen zu beobachten als ideologische Reaktion auf das Phänomen der globalen Kulturindustrie, die 
die kollektive Identität einzelner Gruppen zu bedrohen scheint (Schiffauer 1997:172ff.). Daher muß bei der „Neu-Konstruktion europäischer Identität im multikulturellen Sinn... die kulturindustrielle Pulverisierung wie die fundamentalistische Zementierung vermieden werden" (Lützeler 1995:103). Bisher, so Lützeler, sei eine europäische Identität leider nicht sehr stark entwickelt. Sie zu schaffen, sei aber notwendig, um den tatsächlich bestehenden internationalen Verflechtungen gerecht zu werden und der kulturellen Diskussion zwischen Globalisierung und nationaler bzw. kontinentaler Identität neue Impulse zu geben.

\section{Aktuelle Tendenzen in der Sprachenpolitik}

Die Sprachenpolitik als eines der wichtigsten Bereiche der Kulturpolitik stellt sich in den einzelnen europäischen Staaten sehr unterschiedlich dar. Sie hängt unter anderem davon $a b$, ob es sich um Länder mit ein- oder mehrsprachiger Bevölkerung handelt. Im ersten Fall förderte der Staat lange Jahre lang ganz selbstverständlich die Kultur der Majorität, die entsprechend auch außenpolitische Verbreitung fand, meint Lützeler (Lützeler 1995:100). Das gelte vorrangig für die Sprache und ihre Verbreitung im Ausland durch Goethe-Institute; Instituts Français oder British Councils etc. Wissenschaftler ebenso wie Lehrer sahen die Situation immer schon eher pragmatisch. Aus ihrer täglichen Erfahrung im Umgang mit multikulturellen Klassen sowie der Unabweisbarkeit multikultureller Bevölkerungsstrukturen vor allem in Großstädten plädieren sie dafür, $\mathrm{da}$ sich bereits die Schulen für die Begegnung mit Menschen anderer Sprache und Kultur öffnen. Damit soll der Unterricht bereits in lebensrelevante Zusammenhänge einüben. Dies sollte günstigerweise im Fremdsprachenunterricht geschehen. Damit würde sich die Vermittlung des Stoffes im Unterricht um das Arrangieren kooperativer Lernsituationen erweitern, die eine Anbindung nach außen haben. Fremdsprachen lehren sollte dadurch um die Dimension Begegnung lehren erweitert werden (Alix 1993:112).

Die auswärtige Kulturpolitik Deutschlands bemüht sich neuerdings zunehmend, von der traditionellen Vermittlung der „eigenen“ Kultur im Ausland abzurücken zugunsten eines Austausches und Dialogs mit Partnern. Das bedingt neue Kulturprogramme im Bereich von Präsentationshilfe und Kulturerhalt sowie eine nachhaltigere ausländische Kulturdarstellung in der Bundesrepublik Deutschland. Gefördert werden nunmehr auch verstärkt Austauschprogramme für Studenten und Wissenschaftler. Zudem werden interkulturelle Erwägungen in der täglichen Praxis der Kulturarbeit im Ausland wichtiger. Die Vermittlung eines umfassenden und wirklichkeitsgetreuen Deutschlandbildes orientiert sich an einem adressatenspezifischen Bedarf und an den kulturellen Besonderheiten der Partner. Das findet seinen Niederschlag auch in der Vermittlung der deut- 
schen Sprache im Ausland, die mit Hilfe besonderer Lehrwerke speziell regional ausgerichtet wird. Auch in den deutschen Auslandsschulen stehen neuerdings bikulturelle Begegnungen im Vordergrund. Ebenso geht es bei der Vermittlung europäischer Kulturpolitik gegenüber anderen Kontinenten darum, die Vielfalt der Erscheinungen nicht zu übertünchen, sondern sichtbar zu machen (Witte 1993:458ff.).

In den Staaten mit offiziell mehrsprachiger Bevölkerung gehört die Sprachenpolitik zu einer der zentralen politischen Aufgaben. Beispielhaft sollen hier die Schweiz sowie Belgien Erwähnung finden.

In der Schweiz zeigt sich die Mehrsprachigkeit stark territorial differenziert. Das liegt daran, daß die Kantone die Kulturhoheit besitzen und die offizielle Unterrichtssprache festsetzen. So kommt es, daß in der Deutschschweiz (16 Kantone) nur deutschsprachige, in der frankophonen Schweiz (5 Kantone) nur französischsprachige und im italophonen Gebiet (ein Kanton) nur italienischsprachige öffentliche staatliche Schulen bestehen. Gegen diesen Zustand gibt es keine lauten Proteste. Man stellt ein eher desinteressiertes Nebeneinander der Sprachgemeinschaften fest, die auch territorial ziemlich klar getrennt voneinander leben. Größere Konflikte sind nach Ehrhard (Ehrhard 1993:279) auch deshalb vermieden worden, weil sich an den sprachlichen Nahtstellen keine weiteren sozialen und kulturellen Konflikte (etwa der Religion oder des sozialen Status) auftun. Allerdings ist auch festzustellen, daß die rätoromanische Sprache faktisch nicht mehr existiert und im offiziellen Umgang das Deutsche (allerdings in seinen alemannischen Dialekten) und das Französische vorherrschen. Der Fremdsprachenunterricht zielt darauf ab, die Sprachen der anderen Territorien zu vermitteln, wobei zusätzlich das Englische angeboten und auch nachgefragt wird.

Aufgrund offenkundiger sozialer Spannungen zwischen der französisch- und der deutschsprechenden Bevölkerung, die zwar die Mehrheit der Bewohner stellt, aber sozial schlechter gestellt ist, hat sich Belgien mit dem Problem der Mehrsprachigkeit viele Jahre lang sehr kontrovers auseinandergesetzt. Das Territorialprinzip wurde schließlich von seiten des Staates durchgesetzt, um endlich eine Befriedung der Konflikte herbeizuführen. Auf diese Weise konnte die Funktionsfähigkeit des kleinen Landes erhalten werden. Das Territorialprinzip wurde nicht nur in den Schulen als verpflichtend eingeführt, sondern darüber hinaus auch in den Betrieben, um die sozialen Spannungen abzubauen. Parallel zur Sprachgesetzgebung wurde ein Föderalisierungs- und Regionalisierungskonzept entwickelt, um die Dominanz einer Sprache zu verhindern. Ziel aller Maßnahmen waren Konfliktvermeidung und Konfliktneutralisierung. Dies scheint in den vergangenen Jahren gelungen zu sein. Die Entemotionalisierung des Sprachenstreits förderte wesentlich ein individuelles Sprachverhalten, das faktisch auf einen Zwei- oder Mehrsprachenerwerb hinausläuft. 
Peter H. Nelde zieht folgendes Fazit (Nelde 1994:37): „Sprache als häufiges Sekundärmerkmal zugrundeliegender sozio-politischer und wirtschaftlicher Konflikte gilt in vielen mehrsprachigen Staaten als Konfliktsymbol schlechthin." Er empfiehlt deshalb an die Stelle einer reinen Verstärkung des Fremdsprachenunterrichts die Entemotionalisierung des Konfliktes zu setzen. Eine „symmetrische Zweisprachigkeit" (ebd.) in den Sprachgrenzgebieten könne dazu ebenso beitragen wie das Kennenlernen der Kultur des Nachbarn, ein Abbau von Vorurteilen und Stereotypen und eine stärkere Berücksichtigung der lokalen und regionalen Besonderheiten. Darin stimmt er mit Raasch überein, der Sprachlehrer auch als Kulturlehrer bezeichnet, "weil sie die denotativen und konnotativen Füllungen von Wörtern kennen, denn sie betreiben ja zusammen mit der Spracharbeit zugleich auch die Landeskunde, Kulturkunde." (Raasch 1999:71). Das heißt, daß das Lernen und Lehren von Sprachen auch sprachlichkulturelle Kompetenz vermittelt. Wer Sprachen beherrscht, verfügt über kulturelles Wissen und vermag das eigene und das Fremde in Beziehung zu setzen.

Deshalb sehen Wissenschaftler im Sprachunterricht nicht (mehr) vorrangig die Vermittlung von Grammatik und Vokabular, sondern von Kommunikation. Hier verbinden sich Fremdsprachenunterricht, Landeskunde, Interkulturalität und aktuelle Linguistik. Lehrende sind damit Experten für einen Unterricht, der die Kommunikation zwischen den Kulturen ermöglicht. Deshalb sollte ihre Ausbildung die dafür notwendigen Voraussetzungen schaffen. Hinzu kommt, daß auch die Wirtschaft zunehmend auf Kenntnisse über die Länder angewiesen ist, in die sie exportiert bzw. in denen sie produziert. Nach Raasch sollte der Sprachenunterricht darüber hinaus die aktuelle Situation von Grenzregionen berücksichtigen und die Chancen ergreifen, die damit verbunden sind (Raasch 1999:73).

\section{Die Situation an der deutsch-polnischen Grenze}

Die deutsch-polnischen Beziehungen in der Grenzregion haben sich seit dem Ende des Zweiten Weltkrieges kontinuierlich positiv entwickelt. Belastet durch die Hypothek der nationalsozialistischen Okkupation Polens, war die Wiederannäherung beider Länder zunächst gekennzeichnet durch die Trennung Deutschlands in den der NATO und der Europäischen Union zugehörenden Westteil sowie den dem Warschauer Pakt angehörenden Ostteil (DDR). Der Grundkonflikt bestand nach 1945 in der strittigen Grenzfrage, das heißt der Anerkennung der sogenannten Oder-Neiße-Linie als durch den Krieg faktisch entstandene neue deutsche Ostgrenze (Bingen 1993:406ff.; Jacobsen 1993:395ff.). Die endgültige Grenzziehung sollte nach den Potsdamer Verträgen von 1945 einer internationalen Friedensregelung vorbehalten bleiben. Der 
polnischen Regierung war natürlich an einer möglichst schnellen Anerkennung ihres Territoriums gelegen, schließlich wurde die Republik im Westen nur teilweise für die im Osten an die Sowjetunion verlorenen Gebiete entschädigt. Daß die Regierung der DDR die neue Grenze bereits 1950 in der Warschauer Deklaration und dem Görlitzer Abkommen anerkannte, war zu diesem Zeitpunkt für Polen wenig beruhigend. Denn zum einen fand die DDR keine allgemeine völkerrechtliche Anerkennung und zum anderen standen beide Länder so stark unter sowjetischem Einfluß, daß von einem freien Entschluß der DDR nicht die Rede sein konnte. In der Folgezeit entspannte sich die Grenzsituation auch nur oberflächlich. Tatsächlich war die Oder zu beiden Seiten von Grenzposten schwer bewacht und ein Kontakt der Bevölkerung blieb im wesentlichen offiziellen Begegnungen vorbehalten. Die auf DDR-Seite arbeitenden polnischen Menschen waren in den DDR-Alltag nicht integriert.

Die Bundesrepublik Deutschland tat sich ihrerseits schwer mit der Anerkennung der politischen Gegebenheiten. Lange Jahre dominierten Vertriebenenverbände die Ostpolitik und riefen weniger die durch die Nationalsozialisten begonnene Vernichtungspolitik im Osten als vielmehr die darauf erfolgte Reaktion der Vertreibung deutscher Bevölkerung aus den Ostgebieten in Erinnerung. Die auf diese Weise wachgehaltene Erwartung der Umkehrung der Verhältnisse und möglichen Rückgewinnung verlorener Territorien stand einer Annäherung der beiden Staaten lange Zeit im Wege. Erst die Ostpolitik Willy Brandts ermöglichte einen Neuanfang. In dem Moskauer und dem Warschauer Vertrag 1970 erkannte die Bundesrepublik Deutschland die Oder-Neiße-Linie als Staatsgrenze an. Mit der deutschen Wiedervereinigung 1989 konnte dann auch eine gesamtdeutsche verbindliche Regelung getroffen werden. Am 18. März und 21. Juni 1990 verabschiedeten die erstmals frei gewählte Volkskammer der DDR und der Deutsche Bundestag gleichlautende Erklärungen, in denen die Absicht bekundet wird, den Grenzverlauf zwischen Deutschland und der Republik Polen endgültig völkerrechtlich zu regeln. Dieser Vertrag wurde am 14. November 1990 in Warschau geschlossen. Ergänzend trat am 17. Juni 1991 der Vertrag über gute Nachbarschaft und freundschaftliche Zusammenarbeit hinzu, der den Beginn einer neuen Epoche in der friedlichen Kooperation der beiden Länder einleitete. Dem Nachbarschaftsvertrag kommt eine wegweisende politische Bedeutung zu, denn in ihm werden konkrete Felder der gemeinsamen Zusammenarbeit abgesteckt. Dazu gehören: regelmäßige Konsultationen auf der Ebene der Regierungschefs und der Minister, gemeinsame sicherheitspolitische Abstimmungen, die explizite deutsche Unterstützung zur politischen und wirtschaftlichen Heranführung Polens an die Europäische Union, die Ausweitung der wirtschaftlichen Zusammenarbeit, die partnerschaftliche Zusammenarbeit zwischen Regionen, Städten, Gemeinden und Gebietskörperschaften, insbesondere im grenznahen Bereich, arbeits- und sozialpolitische sowie wissenschaftlich-technische Zusammenarbeit. 
Das neue Verhältnis zwischen Polen und Deutschland wurde wesentlich mit beeinflußt durch die Auflösung der Sowjetunion und des militärischen Bündnisses des Warschauer Paktes. Seither kann sich an der östlichen Grenze Deutschlands eine internationale und interkulturelle Nachbarschaft entwickeln, wie sie sich Jahre zuvor an den Westgrenzen bereits erfolgreich ausgebildet hat. Befördert wird diese neue Annäherung durch die Erwartungen Polens, aufgrund der im Nachbarschaftsvertrag vereinbarten Unterstützung Deutschlands in absehbarer Zeit vollwertiges Mitglied in der Europäischen Union zu werden. Um die Aufnahmekriterien zu erfüllen, bedarf es zur Zeit noch zahlreicher struktureller Anpassungen, zu denen vorrangig eine stärkere Dezentralisierung der Verwaltung und damit verbunden die Schaffung regionaler Entscheidungsebenen gehört. In diesem Bereich wurden bereits wesentliche Schritte unternommen, nicht zuletzt die Gründung von drei Euroregionen an der Grenze zu Deutschland, die geeignet sind, das Zusammenwachsen der Regionen über die Grenze hinaus zu beflügeln. Die Grundidee der Euroregionen besteht darin, die trennende Wirkung von Staatsgrenzen sowie Entwicklungsdisparitäten zwischen benachbarten Teilregionen von Grenzgebieten durch politische Vernetzung beiderseits der Grenze abzubauen, um die Lebensverhältnisse in den genannten Regionen zu vereinheitlichen. Die Euroregionen Pomerania, ProEuropa-Viadrina und Spree-Neiße-Bober (von Norden nach Süden) wurden seit 1992 über den gesamten Grenzverlauf zwischen der Republik Polen und der Bundesrepublik Deutschland eingerichtet.

Wie bereits erwähnt, konnte sich an der Grenze von Oder und Neiße nach den gewaltigen Bevölkerungsverschiebungen im Zuge des Zweiten Weltkrieges keine Tradition des friedlichen Miteinanders der Bevölkerungen entwickeln. Zum einen fand ein fast völliger Austausch der Bewohner statt, hervorgerufen durch eine allgemeine Westverschiebung der Bevölkerung. Zum anderen kam es zu einer konsequenten und hermetischen Abriegelung der Staatsgrenzen, die auch eine sprachliche Annäherung der Nachbarstaaten weitgehend verhinderte. Darüber hinaus muß ein deutliches Wohlstandsgefälle zwischen den beiden Grenzgebieten festgestellt werden. (Davon ist nicht berührt, daß wiederum innerhalb Polens die westlichen Gebiete deutlich wachstums- und wirtschaftsstärker sind als die Gebiete an der Ostgrenze.) Für die neuen deutsch-polnischen Euroregionen gelten daher besondere Bedingungen, die nach dem Ende des Kalten Krieges und der Transformation der Mitteleuropäischen Staaten eine eigene Entwicklungschance beinhalten. Als Brücke zwischen Deutschland und Polen sowie zwischen der EU im Westen und den neuen parlamentarischen Demokratien im Osten kommt dem unmittelbaren Grenzbereich eine neue wichtige politische Aufgabe zu. Die grenzüberschreitende Zusammenarbeit lohnt sich für die lokalen Akteure auch wirtschaftlich, da die Europäische Union mit zahlreichen Förderinstrumenten diese Zusammenarbeit über die Grenze hinweg 
unterstützt. Die Euroregionen können sich organisatorisch an die bereits etablierten Einrichtungen in Nord- und Westeuropa anlehnen und auf diese Weise von bereits langjährig erfolgreichen Strukturen profitieren. Trotz bestehender Konflikte haben die Kommunen die Gründung der Euroregionen forciert, um mittel- und langfristig politisch und wirtschaftlich verbesserte Beziehungen aufzubauen. Nicht zu verkennen ist natürlich, daß das positive Entwicklungspotential der intensiven Pflege und Unterstützung bedarf. Die Lösung gemeinsamer Probleme und die Verwirklichung gemeinsamer Entwicklungsstrategien hängt sehr stark von den lokalen und regionalen Akteuren $a b$, die darüber hinaus der politisch-rechtlich-finanziellen Unterstützung der EU-Kommission bedürfen. Dazu wurden inzwischen die institutionellen Rahmenbedingungen von seiten der Gemeinschaft und der beiden betroffenen Staaten geschaffen. $\mathrm{Da}$ sie zumindest schon ansatzweise genutzt werden, zeigt die Entwicklung an der Grenze selbst: der Bau gemeinsamer infrastruktureller Einrichtungen, die Investitionen großer Industriekonzerne etc. Nicht zu vergessen ist, daß die Bundesrepublik Deutschland als größter Exporteur von Kapitalgütern in der Union von dem anhaltenden Wirtschaftswachstum in den Reformländern Mittelosteuropas besonders stark profitieren wird.

Die Entwicklung der Euroregion Pro Europa Viadrina kann dies beispielhaft belegen (Morhard 2001:164ff.). Der Gründungsvertrag für diese Euroregion wurde Ende 1993 geschlossen und beinhaltet, ein gutnachbarschaftliches Verhältnis, die Unterstützung der regionalen Identität der im Grenzraum lebenden Bevölkerung, die Idee der europäischen Einheit und Verständigung, den Wohlstand beiderseits der Grenzen zu fördern und gemeinsame Vorhaben durchzufüren. Die Euroregion reicht von Berlin im Westen bis zur Grenze der polnischen Wojewodschaft Lubuskie (Lebuser Land, früher: Gorzowskie) im Osten. Dazu zählen auf polnischer Seite 29 Gemeinden und auf deutscher die beiden Landkreise Märkisch-Oderland und Oder-Spree sowie die kreisfreie Stadt Frankfurt (Oder). Die Euroregion wird durch zwei Vereine, ihre Geschäftsstellen sowie kommunale Gebietskörperschaften, Kammern und Parlamente institutionalisiert, aus deren Mitte ein vierköpfiges Präsidium gewählt wird. Die Präsidentschaft wechselt jährlich zwischen dem deutschen und dem polnischen Vertreter. Zu den wichtigsten Aufgaben der Gremien zählen Projektanträge die Verwaltung der von der Europäischen Union bereitgestellten Mittel aus den Förderprogrammen. Das Interreg-Programm der Europäischen Union besteht seit 1990 und zielt darauf ab, den Grenzregionen bei der Bewältigung besonderer Entwicklungsprobleme zu helfen. Im Zuge der Unterstützung des demokratischen und marktwirtschaftlichen Reformprozesses in Mittel- und Osteuropa wurde außerdem bereits 1989 die Initiative für das Phare-Programm ergriffen, das sich zunächst nur auf Polen und Ungarn 
bezog, inzwischen aber auch auf weitere Reformstaaten in Ost- und Mitteleuropa ausgedehnt wurde.

Die Gewährung von Phare-Mitteln ist an die Erfüllung von bestimmten Rahmenbedingungen, wie die Beachtung der Menschenrechte, Rechtsstaatlichkeit u.s.w. gebunden. Sie sollen den Reformstaaten bei der Umgestaltung von Staatsbetrieben und der Entwicklung eines Privatsektors, der Reform von Verwaltung und Landwirtschaft, dem Gesundheitswesen, der Ausbildung, der Umwelt und nuklearen Sicherheit helfen. Ergänzt wird das Phare-Programm seit 1994 durch die zusätzliche grenzübergreifende Fördermöglichkeit CBC (Cross-BorderCooperation). Aus den EU-Programmen Interreg II und Phare-CBC wurden in der Region Pro Europa Viadrina zum Beispiel folgende grenzüberschreitenden Projekte unterstützt: Umweltbericht der Städte Frankfurt (Oder) und Słubice, das Laser- und Strahltechnik Applikationszentrum Ostbrandenburg, Hafenverbünde, Fernradwanderwege, das Collegium Polonicum als eine gemeinsame Einrichtung der Europa-Universität Viadrina Frankfurt (Oder) und der Adam-MickiewiczUniversität in Poznań/Posen, das Zollabfertigungsterminal in Świecko II, die Modernisierung von Straßen sowie drei kommunale Kläranlagen.

\section{Die Bedeutung der Nachbarsprachen an der deutsch-polnischen Grenze}

Sprachliche Beziehungen zwischen Deutschen und Polen bestehen seit dem Mittelalter (de Vincenz 1993:395ff.). Von der räumlichen Nähe der damals slawischen und germanischen Bevölkerung im polnisch-deutschen Raum legen bis heute etymologische Entsprechungen Zeugnis ab. Die kulturelle Nähe der Völker entstand durch die Christianisierung Polens im 10. Jahrhundert nach Christus. Die Missionare gehörten zur Diözese Regensburg und brachten einen noch karolingischen Wortschatz mit. Vielfach gingen in dieser Zeit deutsche und natürlich auch lateinische Lehenswörter in den polnischen Wortschatz über. Im Zuge der deutschen Ostkolonialisierung weitete sich der deutsche Einfluß sowohl rechtlich-politisch (Magdeburger Stadtrecht) sowie dadurch bedingt auch sprachlich weiter aus (Beispiel: burmistrz/Bürgermeister, ein sowohl aus dem Lateinischen wie dem Deutschen entlehnter Begriff). Lehenswörter lassen sich vor allem im religiösen, ritterlichen und städtischen Bereich nachweisen.

Weiteren Einfluß auf die polnische Sprache gewannen im 16. bis 18. Jahrhundert das Italienische und Französische aufgrund ihrer kulturellen bzw. politischen Dominanz in Europa. Zwar ging das Polnische immer mehr in die Umgangs- und Schriftsprache ein. Doch blieb die deutsche Sprache in den überwiegend deutschsprechenden Städten und Provinzen erhalten: Westpreußen, Schle- 
sien, Danzig. Die Wirkung des Protestantismus in einigen polnischen Regionen ebenso wie eine ausgedehnte Wanderhandwerkerschaft von West nach Ost förderten ebenfalls den Gebrauch der deutschen Sprache und schlugen sich in zahlreichen noch heute festzustellenden Lehenswörtern nieder, wie zum Beispiel das Wort druk für Druck, malarz für Maler, landszafi für Landschaft u.s.w. Verstärkt wurden diese Strömungen nach den polnischen Teilungen, als große Gebiete des Landes unter preußischer bzw. österreichischer Verwaltung gerieten.

Umgekehrt ist der Einfluß der polnischen auf die deutsche Sprache nicht in gleicher Weise umfangreich gewesen, was sicherlich mit der geopolitischen und historischen Entwicklung in Mitteleuropa zusammenhängt. Immerhin, es gibt polnische Lehenswörter im Deutschen, wie das wichtigste von ihnen: granica Grenze beweist (erstmals erwähnt um 1260 in Urkunden des Deutschen Ordens). Andrzej de Vincenz wertet die Befruchtung der polnischen durch die lateinischfranzösisch-deutschen Einflüsse insgesamt positiv (de Vincenz 1993:122), bedeute sie doch eine Offenheit der Kultur ohne Aufgabe der eigenen Identität.

Nach dem Zweiten Weltkrieg stellte sich die Situation plötzlich anders dar. Denn aufgrund der politischen Situation gelangten weder das Polnische noch das Deutsche im jeweiligen Nachbarland zu neuer Blüte. Vielmehr wurden die Staaten des Warschauer Paktes politisch und sprachlich durch das Russische bzw. durch die Sowjetunion dominiert. Im Ostdeutschen hat dies bis heute Spuren hinterlassen durch russische Lehenswörter, die Eingang in die Umgangssprache und mehr noch in die offizielle Sprache fanden: Ukas (Verordnung), Kolchose, Datsche etc. Da andererseits der sowjetische Einfluß in den Ländern des Warschauer Paktes weitgehend als fremdbestimmt galt, wurde die russische Sprache nach 1989 zunächst nicht mehr weiter gepflegt, sondern im Gegenteil zumindest vorübergehend schnell vergessen. Bis heute erscheint es ratsam, sich des Russischen im höflichen Umgang im Baltikum ebenso wie in Polen nicht zu bedienen, da diese Sprache als Idiom der Unterdrücker immer noch lebendig ist.

Wenn also das Russische als übergreifendes Verständigungsmittel entfällt, bedarf es heute neuer Instrumente der Verständigung. Denn wirtschaftliche wie auch politische Zusammenarbeit können ohne gemeinsame Kommunikationsebene nicht erfolgreich vonstatten gehen. So wurden in den letzten Jahren in den Grenzregionen beiderseits von Oder und Neiße verstärkt Anstrengungen unternommen, die bestehenden Sprachbarrieren zu überwinden und die Nachbarsprache zu erlernen. Auf polnischer Seite stellt sich das Problem sicherlich nicht so gravierend dar, weil im Vorfeld eines EU-Beitritts Polens zum einen das Interesse an der Nachbarsprache bei jungen Polen geweckt wurde. Zum anderen wird der deutsche Besucher inzwischen in den touristischen Regionen vielfach in seiner Landessprache angeredet, woraus ein wesentlicher Impuls für die weitere wirtschaftliche Entwicklung des Landes erwächst. Auf deutscher Seite haben sich vor 
allem die Urania und die Volkshochschulen für eine Verbreitung der polnischen Sprache eingesetzt. Thre Sprachkurse richten sich in erster Linie an Arbeitnehmer, die beruflichen Kontakt nach Polen haben. Des weiteren wurden mit Mitteln der Europäischen Union zahlreiche grenzübergreifende Bildungsprojekte angestoßen, von denen an dieser Stelle die deutsch-polnischen Schulprojekte im Land Brandenburg, in Sachsen, Mecklenburg-Vorpommern und Bayern mit polnischen Partnern genannt seien.

In Brandenburg läuft das Experiment der deutsch-polnischen Schulpartnerschaften mit dem Abschluß des deutschen Abiturs seit dem Schuljahr 1992/93 (Morhard 2001:214ff.). Ziel dieser von Eltern, Schülern und Lehrern beiderseits der Grenze ergriffenen Initiative ist es, „Kinder und Jugendliche im Sinne gegenseitiger Toleranz zu bilden und zu erzieheiı" (Morhard 2001:215) und im grenznahen Raum die Verständigung untereinander zu fördern. Die Partnerschulen in Brandenburg sind: Frankfurt (Oder) - Słubice, Guben - Gubin, Neuzelle - Zielona Góra sowie Gartz - Gryfino. Auswahlkriterien in Polen sind sehr gute Deutsch- und (falls notwendig) ausreichende Fachkenntnisse. Ziel der Zusammenarbeit ist die Erlangung des bundesdeutschen Abiturs. Das Land Brandenburg unterstützt die polnischen Partner beim Deutschunterricht. Hintergrund des Projektes ist die Tatsache, daß bis heute die polnische Matura allein noch nicht als Zulassungsberechtigung für ein Studium in der EU anerkannt ist. Nach den bisherigen Erfahrungen sind die deutschen Lehrkräfte von der Motivation und Leistung ihrer polnischen Schüler beeindruckt. Wenn auch nicht im gleichen Umfang Polnisch an den deutschen Schulen unterrichtet wird, ist doch festzustellen, daß sich die Partnerschulen um den kulturellen Austausch von Sprache und Kultur in beiden Richtungen bemühen. Zum Beispiel werden in der Partnerschule in Frankfurt (Oder) ab der 9. Klasse Polnisch unterrichtet sowie die Fächer Kunst und Musik in polnischer Sprache vermittelt. Langfristig planen die Schulen einen Ausbau ihres zweisprachigen Lehrangebotes mit dem Ziel, bilingual zu werden. Bislang ist dieses Projekt weitgehend auf die deutsch-polnische Grenzregion beschränkt. Allerdings hat das Berliner Erich-Fried-Gymnasium Interesse bekundet, eine „deutsch-polnische Europaschule ${ }^{\text {“ }}$ zu werden.

Weitere Kooperationsprojekte im Bildungsbereich der brandenburgischpolnischen Grenze ist die Partnerschaft zwischen den allgemein- und berufsbildenden Schulen in Schwedt und Chojna durch "reisende Lehrkräfte“. An zwei weiteren brandenburgischen Grundschulen fördert die Europäische Union das Projekt „Begegnungssprache“ zwischen Schülern in drei Klassen in Polnisch. Für jeweils rund 500 Schüler auf polnischer und deutscher Seite unterstützt die Europäische Union seit 1994 Arbeitsgemeinschaften an Grundschulen in Brandenburg. Des weiteren existieren Begegnungsprojekte im Vorschulbereich, zum Beispiel eine deutsch-polnische Kindertagesstätte in Eisenhüttenstadt. 


\section{Die Rolle der Europa-Universität Viadrina Frankfurt (Oder) im Prozeß der Annäherung von Polen und Deutschen}

In dem Bemühen sowohl der Europäischen Gemeinschaft als auch der beiden Staaten Polen und Deutschland um Annäherung und Zusammenarbeit kommt der 1991 wiederbegründeten Viadrina Universität in Frankfurt (Oder) eine zentrale Rolle zu. In diesem Zusammenhang muß auch der von der Viadrina und ihrer Partneruniversität in Poznań (Posen) geleistete Aufbau des Collegium Polonicum, einer gemeinsamen Lehr- und Forschungseinrichtung in Słubice, dem Frankfurt (Oder) gegenüberliegenden polnischen Grenzort, erwähnt werden.

Schon die kurfürstliche brandenburgische Regierung errichtete die erste Landesuniversität im Jahre 1506 an der Oder. Vor allem Studenten aus dem mittleren und östlichen Europa nahmen hier ihr Studium der Theologie, Philosophie, Rechtswissenschaft oder Medizin auf. Insgesamt wurden 25 Disziplinen gelehrt, unter ihnen Rhetorik, Geschichte, Astronomie, Mathematik und Musik. Als Begegnungsstätte zwischen Ost und West machte sich die Oderuniversität früh einen Namen (Weiler 1996:9f.). Frankfurt (Oder) wurde für junge Leute aus dem märkischen, pommerschen, lausitzer, schlesischen und großpolnischen Umland sowie dem entfernteren Osten häufig die erste Station ihrer Studien, von wo aus sie später nach Jena oder Wittenberg weiterreisten. Die Universität vertrat bekanntermaßen die Ideen der Reformation, so daß sie als Rückhalt, Sammel- und Ausstrahlungspunkt der Aufklärung galt und als ein (von Westen aus gesehen) östlicher Vorposten fortschrittlicher Lehren. Zu ihren bekanntesten Studenten zählten Ulrich von Hutten, Thomas Müntzer, Carl Philipp Emanuel Bach, Wilhelm und Alexander von Humboldt sowie Heinrich von Kleist. Die alte Viadrina wurde zu einer Drehscheibe des akademischen Verkehrs zwischen dem westlichen und östlichen Europa und spielte die Rolle einer Vermittlerin der geistigen und demographischen Wanderungsbewegungen zwischen Westund Osteuropa (die Hugenotten von West nach Ost, der polnische Adel von Ost nach West). Diese Entwicklung brach plötzlich ab, als die preußische Regierung 1910 in ihrer Hauptstadt Berlin eine neue Universität gründete und die Viadrina im darauffolgenden Jahr schloß. Die Viadrina wurde nominell mit der Breslauer Universität Leopoldina vereinigt, ihr Inventar und auch einige ihrer Gelehrten siedelten dorthin über.

Von ihrer historischen Vorgängerin sowie der exponierten Lage an der Grenze der Europäischen Union blieb die neue Viadrina nicht unbeeinflußt. Nach den Vorstellungen der Landesregierung von Brandenburg und des Gründungssenats der Universität sollte sich die 1991 errichtete „Europa-Universität“ durch ein in zweifacher Hinsicht unverwechselbares Profil in Forschung und Lehre auszeichnen: hohe Internationalität sowie Interdisziplinarität (Morhard 
2001:210ff.; Weiler 1996:10). Es galt also, Grenzen in mehrfacher Hinsicht zu überschreiten: räumlich und fachlich. So gesehen ist die Viadrina bis heute einer der wenigen Versuche, das im Westen Deutschlands entwickelte Hochschulmodell nicht einfach zu übertragen, sondern neue Wege der Hochschulentwicklung zu erkunden: Die Viadrina ist daraufhin konzipiert, als bewußt internationale und europäische Einrichtung den geistigen Brückenschlag zu ihren europäischen Nachbarn zu suchen und gleichzeitig einen Beitrag zur Überwindung der Isoliernng der Fachwissenschaften voneinander zu leisten. Letzteres bedeutet, $\mathrm{da} B$ die drei Fakultäten der Rechtswissenschaft, der Kulturwissenschaft und der Wirtschaftswissenschaften darauf hinarbeiten, auch für die Nachbardisziplinen Angebote zu schaffen.

Die Basis der Zusammenarbeit bildete zunächst eine Vereinbarung zwischen dem polnischen Ministerium für Nationale Bildung und dem brandenburgischen Ministerium für Wissenschaft, Forschung und Kultur vom 6. September 1991, dem sich am 24. März 1992 ein Partnerschaftsvertrag zwischen der Adam-Mickiewicz-Universität in Poznań (Posen) und der Europa-Universität anschloß. In den folgenden Jahren wurden weitere Abkommen mit den Universitäten in Wrocław (Breslau), Szczecin (Stettin), Kraków (Krakau) und Warszawa (Warschau) geschlossen. Alle Vereinbarungen haben konkrete Formen der Zusammenarbeit gezeitigt. Die universitären Partnerschaften werden von seiten der Landesregierung positiv begleitet, auf Regierungsebene liegt die Koordination der wissenschaftlichen Zusammenarbeit in den Händen eines brandenburgisch-polnischen Koordinationsgremiums auf Abteilungsleiterebene.

Der folgenreichste Schritt der Zusammenarbeit bestand auf deutscher Seite in der Entscheidung, rand ein Drittel der Studienplätze an der Viadrina für polnische Studierende zu reservieren (sowie für Studierende aus weiteren MOELStaaten), und zwar nicht nur im Rahmen von Gastaufenthalten, sondern für die gesamte Dauer eines Studiums. Von den im Wintersemester 2000/2001 immatrikulierten rund 3.800 Studierenden der Viadrina waren rund 1.350 polnische Studierende. Rund 200 weitere ausländische Studierende kamen aus 46 Ländern der ganzen Welt. Die polnischen Studierenden werden vorab von den Partneruniversitäten in Posen und Breslau ausgewählt, müssen sich allerdings dann noch einer besonderen Deutschprüfung unterziehen.

Das Studium in Deutschland bedeutet für Studierende aus den Ländern Ostmitteleuropas einen beträchtlichen zusätzlichen finanziellen Aufwand (Weiler 1996:10f.). Deshalb gehört zu dem Konzept der Europa-Universität, den bedurftigen Studierenden aus diesen Ländern die finanzielle Mehrbelastung in Form von Stipendien zumindest teilweise auszugleichen. Daraus resultiert ein Mittelbedarf von weit mehr als zwei Millionen DM im Jahr. Einen Großteil des Geldes stellt das Land Brandenburg zur Verfügung. Darüber hinaus engagieren sich inzwischen auch das Auswärtige Amt, die Stiftung für deutsch-polnische 
Zusammenarbeit sowie zahlreiche private Spender in diesem anspruchsvollen Stipendienprogramm.

Ein wichtiger Schwerpunkt der Integration von deutschen und polnischen Studierenden bedeutet die Bereitstellung genügend bezahlbarer studentischer Wohnungen. Mit Hilfe der polnischen Regierung, der Europäischen Union sowie der Posener Partneruniversität entstanden in den letzten Jahren zahlreiche gut erreichbare Wohnheime im polnischen Stubice. Hier wohnen, lernen und feiern polnische, deutsche und andere ausländische Studierende gemeinsam. Das ist übrigens auch in den Wohnheimen auf deutscher Seite möglich. Der dortige Wohnraum wird ebenfalls international genutzt, allerdings sind die Preise nicht so erschwinglich wie auf der anderen Oderseite.

Mit der Öffnung der Universität für eine wirklich bedeutende Anzahl polnischer und weiterer ausländischer Studierender besteht zugleich die Notwendigkeit, Studienangebote bereitzustellen, die den besonderen Interessen und Bedürfnissen dieser Studierenden Rechnung tragen. Beispielhaft kann hier das Studium der Rechtswissenschaft genannt werden. Es hätte sicherlich wenig Sinn, ausländische Studierende nach dem deutschen Richtergesetz auszubilden. Deshalb wurde speziell für polnische Studierende ein Studiengang entwickelt, der eine solide Grundlage im deutschen Recht mit einer in Polen anerkannten und gemeinsam mit der Adam-Mickiewicz-Universität verantworteten Ausbildung im polnischen Recht verbindet. Nach einem ersten Studienabschluß besteht außerdem für ausländische Juristen die Möglichkeit, eine Zusatzqualifikation im deutschen Recht zu erwerben (Magister legum). Zusätzlich arbeitet die Juristische Fakultät an einem Lizentiat-Studiengang „Polnisches Recht für deutsche Studierende". Eine Betonung der internationalen Ausrichtung der Universität besteht auch in den beiden anderen Fakultäten, so im Fach Internationale Betriebswirtschaftslehre der Wirtschaftswissenschaftlichen Fakultät, die außerdem mit verschiedenen Partneruniversitäten im Ausland (Frankreich, Spanien, Argentinien, England) Doppel- bzw. Tripeldiplomierungsabkommen geschlossen hat. Der Nachweis von Kenntnissen in drei lebenden Fremdsprachen ist Voraussetzung für die Anmeldung zur Diplomprüfung in der Kulturwissenschaftlichen Fakultät, darüber hinaus ein Studien- bzw. Praktikumsaufenthalt im Ausland. Da alle Fakultäten Wert auf umfangreiche Fremdsprachenkenntnisse der Studierenden legen, kommt der Arbeit des Sprachenzentrums der Universität große Bedeutung zu. Das Angebot reicht von dem besonders nachgefragten Englisch über Französisch, Spanisch, Russisch, Finnisch und Schwedisch. Inzwischen werden in Zusammenarbeit mit der Urania zwei Italienischkurse angeboten sowie für die ausländischen Studierenden auch ein Kursus Deutsch als Fremdsprache.

Gut bewährt hat sich in den letzten Jahren die Beteiligung der Viadrina an den europäischen Austauschprogrammen für Studierende und Dozenten. Im 
Rahmen von Sokrates/Erasmus können die Studierenden bis zu einem Semester lang an einer europäischen Partneruniversität studieren. Auch den polnischen Viadrina-Studierenden bietet sich dadurch die Möglichkeit, weitere westeuropäische Universitäten im Rahmen ihres Studiums kennenzulernen. Darüber hinaus ermöglichen zahlreiche über die Sokrates-Abkommen hinausgehende Vereinbarungen mit Hochschulen in Europa, Australien, den USA, Mittel- und Südamerika und Afrika den internationalen Austausch von Studierenden und Dozenten. Zur Erleichterung des internationalen Studierendenaustausches hat die Universität das Credit-Transfer-System (ECTS) in den bereits bestehenden grundständigen Studiengängen eingeführt. Auf der Basis von ECTS können im Ausland erbrachte Studienleistungen bewertet und für das heimische Studium angerechnet werden.

Die Europa-Universität hat sich von Beginn an bemüht, ein internationales Lehrkollegium zu versammeln. Wenn auch derartigen Berufungen in Deutschland zahlreiche bürokratische Hindernisse entgegenstehen, konnte die Viadrina letztendlich ihren Anspruch einigermaßen erfüllen. Die Hochschullehrer kommen aus Polen, Australien, Finnland, den Niederlanden, der erste (inzwischen emeritierte) Rektor der Europa-Universität war amerikanischer Staatsbürger. Mit der Verpflichtung ausländischer Kollegen verbindet die Viadrina die Erwartung, neue Impulse für die Forschung und Lehre zu gewinnen und die Studierenden an den internationalen Erfahrungen der Lehrenden teilhaben zu lassen. Gleichzeitig eröffnen sich so neue Perspektiven, Kontakte zu ausländischen Hochschulen zu knüpfen und das bereits bestehende umfangreiche Netz der Partnerschaften noch auszuweiten.

Den Kristallisationspunkt des besonderen internationalen Profils der Europa-Universität bildet zweifellos das gemeinsam mit der Adam-MickiewiczUniversität gegründete und betriebene Collegium Polonicum. Diese einzigartige Einrichtung wirkt bereits optisch imposant. Den aufwendigen Neubau unmittelbar hinter der polnischen Grenze, dicht an der Oder gelegen, haben die Europäische Union und die Stiftung für deutsch-polnische Zusammenarbeit großzügig gefördert. Die polnische Regierung sowie auch die Stadt Słubice haben jeweils auf ihre Weise das Projekt materiell und ideell unterstützt. Beide Universitäten tragen das Projekt in gemeinsamer Verantwortung. Sie verfolgen mit dem Collegium Polonicum die Förderung der Europa-Idee, die Ergänzung des Forschungs- und Lehrangebotes der beiden Universitäten an einem gemeinsamen Ort sowie die Entwicklung und Unterstützung grenzüberschreitender Zusammenarbeit.

Zu den Schwerpunkten in Forschung und Lehre zählen die Geschichte der deutsch-polnischen Beziehungen, polnische Sprache und Kultur, regionale Integration, regionale Umweltforschung sowie die nordosteuropäischen Integrationsprozesse. Bereits seit dem Wintersemester 1993/94 betreibt die Juristische 
Fakultät am Collegium Polonicum unter Einbeziehung polnischer Kollegen die Ausbildung der polnischen Jurastudenten in polnischem Recht. Inzwischen konnte das Studienangebot beider Universitäten am Collegium Polonicum beträchtlich erweitert werden (Morhard 2001:213). Von seiten der EuropaUniversität werden bislang ein Masterstudiengang Vergleichende Mitteleuropastudien, ein MBA-Studiengang Management und Marketing für Mittel- und Osteuropa sowie ein Aufbaustudiengang Schutz europäischer Kulturgüter verantwortet. Außerdem werden zahlreiche Fremdsprachenkurse angeboten. Die Adam-Mickiewicz-Universität betreibt einen grundständigen Studiengang Umweltschutz sowie Angebote in Politologie. Darüber hinaus leitet das Collegium Polonicum selbst ein TEMPUS-Projekt zur Weiterbildung von Städteplanern unter dem Titel „Modernes Stadtentwicklungsmanagement“.

Finanziert werden die von der Viadrina betriebenen Projekte zu einem wesentlichen Teil aus den Interreg-Mitteln der Europäischen Union. Darüber hinaus hat das Bundesbildungsministerium einen Antrag der Universität zum Aufbau eines Graduiertenprogramms inzwischen bewilligt, und das Land Brandenburg will am Collegium Polonicum ein Institut zur Osterweiterung der Europäischen Union einrichten. Die politische Absicherung des Collegium Polonicum soll mit Hilfe eines Staatsvertrages zwischen der Republik Polen und dem Land Brandenburg unter Beteiligung des Auswärtigen Amtes der Bundesregierung erfolgen. Dazu wurden inzwischen die notwendigen Voraussetzungen und Absprachen getroffen, so daß eine Unterzeichnung dieses für die EuropaUniversität so wichtigen Dokumentes alsbald vollzogen werden kann.

\section{LITERATUR}

Alix, Christian (1993): Fremdsprachen lehren und Begegnung lernen: Einige Überlegungen zur Spracharbeit im europäischen Kontext. In: Albert Raasch (Hg.): Fremdsprachen für die Zukunft - Nachbarsprachen und Mehrsprachigkeit. Saarbrücken (Universität Saarbrücken), S. 109-119.

Bingen, Dieter (1993): Oder-Neiße-Linie. In: Ewa Kobylinska u.a. (Hg.): Deutsche und Polen. 100 Schlüsselbegriffe. München (Serie Piper) 2. Aufl., S. 406-413.

Ehrhard, Peter Alex (1993): Mythos, Wirklichkeit und Vision: Vom Umgang mit der sprachlichen und kulturellen Vielfalt in der Schweiz. In: Albert Raasch (Hg.): Fremdsprachen für die Zukunft - Nachbarsprachen und Mehrsprachigkeit. Saarbrücken (Universitat Saarbrücken), S. 275-293.

Finkenstaedt, Thomas; Schröder, Konrad (1990): Sprachenschranken statt Zollschranken? Grundlegung einer Fremdsprachenpolitik für das Europa von morgen. Essen (Stifterverband fur die Deutsche Wissenschaft).

Jakobsen, Hans-Adolf (1993): Deutsche und Polen 1945-1991. In: Ewa Kobylinska u.a. (Hg.): Deutsche und Polen. 100 Schlüsselbegriffe. München (Serie Piper) 2. Aufl., S. 395-401. 
Lützeler, Paul Michael (1995): Vom Ethnozentrismus zur Multikultur. Europäische Identität heute. In: Michael Kessler, Jürgen Wertheimer (Hg.): Multikulturalität. Tendenzen, Probleme, Perspektiven. Tübingen (Stauffenburg Verlag), S. 91-105.

Morhard, Bettina (2001): Das deutsch-polnische Grenzgebiet als Sonderfall europäischer Regionalpolitik. Berlin, Heidelberg u.a. (Springer Verlag).

Nelde, Peter H. (1994): Mehrsprachigkeit und Nachbarsprachen in Europa am Ende des zwanzigsten Jahrhunderts. In: Bernd Spillner (Hg.): Nachbarsprachen in Europa. Frankfurt/Main (Peter Lang Verlag), S. 27-38.

Raasch, Albert (1999): Grenzenlos - durch Sprachen. In: Hans-Jürgen Krumm (Hg.): Die Sprachen unserer Nachbarn - unsere Sprachen. Dokumentation eines Symposiums. Wien 29-31.10.1998. Wien (Wiener Verlags Werkstatt), S. 63-77.

Rosenberg, Peter (1994): Nachbarsprachen in West- und Osteuropa: Einleitendes zum zentralen Themenbereich der GAL 1992, ,Nachbarsprachen im Kontakt‘. In: Bernd Spillner (Hg.): Nachbarsprachen in Europa. Frankfurt/Main (Peter Lang Verlag), S. 39-43.

Schiffauer, Werner (1997): Fremde in der Stadt. Frankfurt/Main (Suhrkamp Taschenbuch 2699).

de Vincenz, Andrzej (1993): Deutsch-polnische Sprachkontakte. In: Ewa Kobylinska u.a. (Hg.): Deutsche und Polen. 100 Schlüsselbegriffe. München (Serie Piper) 2. Aufl., S. 114-122.

Wandruszka, Mario (1994): Europäische Nationalsprachen, Nachbarsprachen, Heimatsprachen. In: Bernd Spillner (Hg.): Nachbarsprachen in Europa. Frankfurt/Main (Peter Lang Verlag), S. 13-27.

Weiler, Hans N. (1996): Wissenschaft an der Grenze. Zum besonderen Profil der EuropaUniversität Viadrina Frankfurt (Oder). Manuskript eines Vortrag an der TU ChemnitzZwickau am 16.1.1996.

Wierlacher, Alois (1993): Xenologie. Ausgangslage, Leitbegriffe und Problemfelder. In: Ders. (Hg.): Kulturthema Fremdheit. Leitbegriffe und Problemfelder kulturwissenschaftlicher Fremdheitsforschung. München (iudicium), S. 19-112.

Witte, Barthold C. (1993): Fremdheitswissen als Basis auswartiger Kulturpolitik, In: Alois Wierlacher (Hg.): Kulturthema Fremdheit. Leitbegriffe und Problemfelder kulturwissenschaftlicher Fremdheitsforschung. München (iudicium), S. 451-461. 